\title{
Coring Techniques for Scientific Investigation of Faults
}

by Dennis L. Nielson

\section{Introduction}

Faulted and fractured rocks are difficult to core because of differential hardness, angular unsupported fragments, under- or over-pressured fluids, and overall fault architecture. Often, the most scientifically interesting parts of the fault are the most difficult to recover. The scientific objectives of the drilling and the techniques required (sampling, logging, monitoring), as well as the depth and geometry of the target, will determine the drilling methods used.

\section{Operational Issues}

The character of fault zones often leads to drilling problems. Due to the abrasive nature of faults, the bit may break or more likely wear on the face or inner and outer gauges. Bits may also plug with a piece of core, or their waterways may become blocked by clay or rock fragments. Cores may jam in the bit or core barrel due to small shifts along fractures during the drilling process. Bits can be custom-designed for specific drilling conditions, and bit life and penetration rate may be improved by size and placement of diamonds, hardness of matrix, and location and geometry of waterways.

Hole stability is a critical factor in operational efficiency; it can be controlled by the circulation of mud whose composition and circulation rate can be optimized. Coring in fault zones often encounters different fluid pressure within short distances. Formation overpressure (greater that hydrostatic) leads to the flow of fluids into the hole, and underpressure results in the loss of circulation in permeable rock. Under conditions where circulation is lost, drilling mud flows into the formation rather than returning to the surface. Cuttings can build up in the hole and stick to the drill pipe. This condition tends to be more serious in rotary drilling than coring, where it is common to core ahead without mud returning to the surface. Lightweight injected grout can be used to shut off lost circulation zones and stabilize fractured rock.

A fault can influence directional control, hole inclination, and doglegs (rapid changes in direction). In general, holes tend to curve into a fault, particularly when the core hole and the fault are both at steep angles. Additionally, the drill bit can become "trapped" within a fault and wander down a soft component, unable to escape. In most cases it is best to plan crossing the fault at as high an angle as possible.

The location of a fault in the subsurface is often uncertain, and intersecting a steeply dipping structure with a vertical borehole is problematic (Are we there yet?). Many coring systems are designed to drill holes at an angle as well as vertically. Directional coring from a borehole is also possible either using a wedge or whip-stock or downhole mud motors. Any of these options allows multiple intersections of a fault to take place from one borehole (multi-laterals).

\section{Core Quality Control}

Core is the principal product of most scientific drilling, and core quality is of utmost concern. High-quality core often requires additional effort, time, and cost. Ideally, the core is a continuous and representative sample of the fault; however, faults are generally broken formations (unless cemented by hydrothermal minerals) and can contain materials of dramatically different hardness. This increases the risk that pieces will be lost or that softer parts of the core may be washed away by the drilling fluid. Once the core is cut, mechanical and thermal stress relief are inevitable and result in the formation of secondary fractures.

Improper core handling can also lead to quality control problems, and it is best to have a well formulated protocol before the drilling phase of the project commences. This is especially important when different people are responsible for handling the core. Problems can result during removal of core from the core barrel, and during boxing, labeling, and transportation.

\section{Bore Hole Quality Control}

The process of drilling changes the borehole environment from in situ conditions. Mechanical disruption results from imposing a hole where there was previously solid rock, and borehole breakouts occur as stress is released. Circulation of drilling mud fills the hole with a fluid that is different from formation fluids in temperature, pressure, and composition. Separate zones that originally had distinct temperature, pressure, and chemistry mix in the borehole. The return to pre-drilling temperatures may require some time (or may never take place). Solids (mud, cement, lost circulation material [LCM]) have probably invaded the rock along faults, 
fractures, and intergranular spaces. Fault heterogeneity can mean that the contamination itself may not be uniform. Chemical and physical tracers can be introduced to assess contamination of the core by the drilling fluid.

\section{Drilling Rigs and Coring Techniques}

Several different types of drilling rigs are available, and they must be chosen to conform to the objectives of the investigation. Rotary rigs are designed to drill a hole with a high penetration rate, and they are principally used in petroleum and groundwater applications. Rotary drilling uses high weight on bit (WOB) and low rotation rate (rpm) in order to crush the rock. High mud volumes are needed to clear the crushed rock from the borehole. Core can be collected by a rotary rig using a conventional barrel, but the quality is subject to the operating parameters of high WOB and low rpm. In addition, the entire drill string must be tripped to recover core from the conventional barrel. This process can be time-consuming, and conventional coring is normally used for spot coring within a zone of interest.

Diamond coring rigs are designed to collect core samples. They do this by cutting the rock with a diamond bit with relatively low and precise control of the WOB and with high rotation rates. Generally, the penetration rate for coring is less than with rotary methods, and the cost/meter can be relatively high. However, the sample is generally superior to that collected by a rotary rig using a conventional barrel. Core is generally collected by wireline systems where the core barrel is retrieved to the surface using a wire while the drilling rods remain in the hole. Wireline coring was developed for mining applications, and coring equipment comes in standard sizes $(\mathrm{PQ}=85 \mathrm{~mm}, \mathrm{HQ}=63.5 \mathrm{~mm}$; $\mathrm{NQ}=$ $47.6 \mathrm{~mm}$ ), although the labels are not particularly intuitive. Larger sizes may be more beneficial in the sampling of faulted rock. A variation on this technique uses a triple tube where the core is collected into a metal or plastic liner that is placed within the core barrel. The core is slightly smaller $\left(\mathrm{PQ}_{3}=83.0 \mathrm{~mm}, \mathrm{HQ}_{3}=61.1 \mathrm{~mm}\right)$, but there is also much less sample disruption.

Slim-hole wireline coring systems are designed to core a telescoped hole where smaller standard sizes are designed to core through larger sizes $(\mathrm{PQ}>\mathrm{HQ}>\mathrm{NQ})$. Therefore, if penetration is stopped, rods can be left in the hole as casing, and the next smaller size system can be used to continue coring.

Because of the need for the characteristics of both rotary and diamond coring, several hybrid rigs have been constructed. These rigs can drill rotary and set casing and then switch to wireline diamond coring for the collection of core samples. Examples of this type of rig include the DOSECC (Drilling, Observation and Sampling of the Earth's Continental Crust) Hybrid Coring System (DHCS) that has been used to core the Long Valley, Hawaii, and Chicxulub Scientific Drilling Projects. The DHCS can attach to most large rotary rigs, thereby limiting its shipping costs. ICDP's new Innova Rig is a self-contained rig that performs both coring and rotary functions.

\section{Concluding Statement}

Faults often have complex histories and, as a result, they show three-dimensional architecture with intricate cross- cutting features. A borehole (core sample) is small, and by itself is largely one-dimensional. Because of the complexity of faults and the quality control issues discussed above, it is advantageous to incorporate logging to support the interpretation of the core. In particular, imaging logs are of great value for their ability to orient fractures in the core and, in some cases, to document material that was lost during the coring process. Temperature logs are also of great value in determining the location and characteristics of zones where fluids are either entering or exiting the borehole. Multiple completions drilled directionally from the same borehole are useful for documenting fault heterogeneity.

\section{Author}

Dennis L. Nielson, DOSECC, Inc., P.O. Box 58857, Salt Lake City, Utah 84158-0857, U.S.A., e-mail: dnielson@ dosecc.org. 